\title{
High Serum CA19-9 Concentration Predicts Poor Prognosis in Elderly Patients with Stage IV Colorectal Cancer
}

\author{
Eiji Hidaka Chiyo Maeda Kenta Nakahara Kunihiko Wakamura \\ Yasuhiro Ishiyama Shoji Shimada Junichi Seki Yojiro Takano \\ Sonoko Oae Yuta Enami Naruhiko Sawada Fumio Ishida Shin-ei Kudo \\ Digestive Disease Center, Showa University Northern Yokohama Hospital, Yokohama, Japan
}

\section{Keywords}

Carbohydrate antigen 19-9 · Stage IV colorectal cancer · Elderly patient

\begin{abstract}
Background/Aim: The optimal treatment strategy for elderly patients with stage IV colorectal cancer (CRC) remains controversial due to limited research data. The purpose of this study was to evaluate treatment results and to clarify the prognostic factors, especially poor prognosis factors, in elderly patients with stage IV CRC. Methods: We retrospectively reviewed the data of 82 elderly patients (aged $\geq 75$ years) with stage IV CRC who underwent surgical treatment at our hospital between April 2001 and March 2017. Factors that affected prognosis and the ability to undergo treatment were analyzed via multivariate analysis. Results: The median overall survival (OS) in the patients with high pretreatment serum carbohydrate antigen 19-9 (CA19-9) concentration (>370 U/mL) was significantly worse than in those with lower serum CA19-9 concentration (0-370 U/mL) (8.5 vs. 19.2 months, $p=0.0059)$. In univariate analysis, age ( $\geq 80$ years) $(p=0.014)$, performance status of $1-3(p=0.028)$, and high pretreatment serum CA19-9 concentration ( $p=0.014$ ) were significant prognostic factors for poor OS. By contrast, resection of the primary tumor ( $p=0.024)$, chemotherapy $(p<0.0001)$, and resection of distant metastasis ( $p=0.0005$ ) were significant prognostic factors for favorable OS. Multivariate analysis showed that a high pretreatment serum CA19-9 concentration was an independent prognostic factor for poor OS ( $p=0.01)$. Meanwhile, resection of the primary tumor $(p=0.033)$, chemotherapy $(p<0.0001)$, and resection of distant metastasis $(p=0.0008)$ were prognostic factors for favorable OS. Conclusions: A high pretreatment serum CA19-9 concentration (>370 U/mL) was a reliable predictive factor for poor prognosis, and aggressive treatments should be performed carefully in these patients. Moreover, various treatments, including surgery and chemotherapy, might improve OS in elderly patients with stage IV CRC.
\end{abstract}




\section{Introduction}

Colorectal cancer (CRC) is one of the most common cancers worldwide [1, 2]. Although the incidence of CRC tends to increase with age [3], most clinical trials have included patients aged $<75$ years, and only few randomized studies on elderly patients (i.e., individuals aged $\geq 75$ years) with CRC have been conducted. Therefore, data on the optimal treatment for elderly patients with CRC have only been derived from retrospective studies [4-8]. As such, the optimal treatment strategy for elderly patients with stage IV CRC is controversial. Some guidelines recommend surgical resection for primary CRC and resectable distant metastatic lesions $[9,10]$. Meanwhile, chemotherapy is recommended for unresectable lesions $[9,10]$. However, these treatments may not yield similar clinical benefits in elderly patients with stage IV CRC because of age-related physiological changes. Considering the average life span of elderly people and the poor prognosis of stage IV CRC [4], especially in elderly patients, it may be necessary to find a balance between palliative care and aggressive treatments. Therefore, it is important to investigate poor prognosis factors to be able to avoid unnecessary aggressive treatments in elderly patients. As such, there is limited knowledge regarding the prognostic factors and optimal treatment strategies in these patients. The purpose of this study was to investigate treatment outcome and determine poor prognostic factors in elderly patients with stage IV CRC.

\section{Patients and Methods}

\section{Patients}

We retrospectively reviewed the data of 82 elderly patients (aged $\geq 75$ years) with stage IV CRC who underwent surgical treatment at Showa University Northern Yokohama Hospital between April 2001 and March 2017. CRC was confirmed via colonoscopy and pathological examination. Distant metastasis was confirmed via computed tomography, magnetic resonance imaging, or positron emission tomography computed tomography, while peritoneal dissemination was diagnosed via intraoperative findings. The clinical and pathological characteristics of the patients, treatment methods, and the overall survival (OS) rate were analyzed retrospectively.

\section{Study Parameters}

The following patient data were collected: age, sex, primary tumor location, Eastern Cooperative Oncology Group performance status (PS), pretreatment concentrations of serum carcinoembryonic antigen (CEA) and serum carbohydrate antigen 19-9 (CA19-9), resection of the primary tumor, emergency operation, lymphatic metastasis, number of metastatic organs (M1a and M1b), severe metastatic status (P3, H3, or PUL2), chemotherapy, and OS. Transverse colon cancer was categorized into the right-sided cancer group. Pretreatment concentrations of serum CEA and serum CA19-9 were divided into three categories, i.e., normal, elevated, and high. The cutoffs for normal, elevated, and high CEA were $<5 \mathrm{ng} / \mathrm{mL}, 5-50 \mathrm{ng} / \mathrm{mL}$, and $>50 \mathrm{ng} /$ $\mathrm{mL}$, respectively, while they were $<37 \mathrm{U} / \mathrm{mL}, 37-370 \mathrm{U} / \mathrm{mL}$, and $>370 \mathrm{U} / \mathrm{mL}$ for CA19-9, respectively. Metastatic organ involvement and severe distant metastasis status were defined according to the 8th edition of the Japanese Classification of Colorectal Carcinoma by the Japanese Society for Cancer of the Colon and Rectum [11]. OS was defined as the interval from the time of primary surgery or primary chemotherapy to the date of death or last follow-up.

\section{Statistical Analysis}

All statistical analyses were performed using the JMP ${ }^{\circledR} 13$ software (SAS Institute Inc., Cary, NC, USA). OS was calculated via the Kaplan-Meier method, and log-rank tests were used for comparison. The Cox regression model was used to determine the impact of selected factors on OS. A Cox proportional hazards model was used to calculate hazard ratios and 95\% confidence intervals for both univariate and multivariate analyses. $p$ values $<0.05$ were considered to indicate significance. 


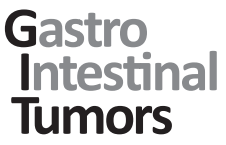

Table 1. Characteristics of the 82 patients with stage IV colorectal cancers

\begin{tabular}{l|l}
\hline Gastrointest Tumors 2018;5:117-124 \\
\hline DOI: 10.1159/000493793 & $\begin{array}{l}\text { @ 2019 S. Karger AG, Basel } \\
\text { www.karger.com/gat }\end{array}$ \\
\hline
\end{tabular}

\begin{tabular}{|c|c|c|}
\hline Characteristics & $n$ & $\%$ \\
\hline \multicolumn{3}{|l|}{ Age } \\
\hline 75-79 years & 43 & 52.4 \\
\hline$\geq 80$ years & 39 & 47.6 \\
\hline \multicolumn{3}{|l|}{ Sex } \\
\hline Male/female & $42 / 40$ & $51.2 / 48.8$ \\
\hline \multicolumn{3}{|l|}{ Primary tumor location } \\
\hline Right/left & $38 / 44$ & $46.3 / 53.7$ \\
\hline \multicolumn{3}{|l|}{ Performance status } \\
\hline $0 / 1-3$ & $57 / 25$ & $70.0 / 30.0$ \\
\hline \multicolumn{3}{|c|}{ Pretreatment serum CEA concentration } \\
\hline Normal $(<5 \mathrm{ng} / \mathrm{mL})$ & 21 & 25.6 \\
\hline Elevated $(5-50 \mathrm{ng} / \mathrm{mL})$ & 32 & 39.0 \\
\hline High $(>50 \mathrm{ng} / \mathrm{mL})$ & 29 & 35.4 \\
\hline \multicolumn{3}{|c|}{ Pretreatment serum CA19-9 concentration } \\
\hline Normal $(<37 \mathrm{U} / \mathrm{mL})$ & 41 & 50.0 \\
\hline Elevated $(37-370 \mathrm{U} / \mathrm{mL})$ & 27 & 32.9 \\
\hline High $(>370 \mathrm{U} / \mathrm{mL})$ & 14 & 17.1 \\
\hline \multicolumn{3}{|l|}{ Resection of the primary tumor } \\
\hline Yes/no & $66 / 16$ & $80.5 / 19.5$ \\
\hline \multicolumn{3}{|l|}{ Emergency operation } \\
\hline Yes/no & $22 / 60$ & $26.8 / 73.2$ \\
\hline \multicolumn{3}{|l|}{ Lymphatic metastasis } \\
\hline $\mathrm{N} 0 / \mathrm{N}(+)$ & $52 / 13$ & $80.0 / 20.0$ \\
\hline \multicolumn{3}{|l|}{ Number of metastatic organs } \\
\hline M1a/M1b & $64 / 18$ & $78.0 / 22.0$ \\
\hline \multicolumn{3}{|l|}{ P3, H3, or PUL2 } \\
\hline Present/absent & $36 / 46$ & $43.9 / 56.1$ \\
\hline \multicolumn{3}{|l|}{ Chemotherapy } \\
\hline Yes/no & $38 / 44$ & $46.3 / 53.7$ \\
\hline \multicolumn{3}{|l|}{ Resection of metastatic tumor } \\
\hline Yes/no & $6 / 76$ & $7.3 / 92.7$ \\
\hline
\end{tabular}

CA19-9, carbohydrate antigen 19-9; CEA, carcinoembryonic antigen.

\section{Results}

The clinical and pathological characteristics of the 82 patients are presented in Table 1. The primary tumor was located on the right and left side in 38 patients (46.3\%) and 44 patients (53.7\%), respectively. The median pretreatment concentration of serum CEA and serum CA19-9 were $25.1 \mathrm{ng} / \mathrm{mL}$ and $32.6 \mathrm{U} / \mathrm{mL}$, respectively. The pretreatment serum CEA concentration was elevated in 61 patients (74.4\%) and high in 29 patients (35.4\%). Meanwhile, the serum CA19-9 concentration was elevated in 41 patients (50.0\%) and high in 14 patients $(17.1 \%)$. A total of 66 patients $(80.5 \%)$ underwent resection of the primary tumor, and 38 patients $(46.3 \%)$ received chemotherapy. Of them, 16 patients $(42.1 \%)$ received oral 5 -fluorouracil (5-FU) anticancer agents, 12 patients (31.6\%) received oxaliplatin or irinotecan combined with the 5-FU regimen, and 10 patients $(26.3 \%)$ received targeted chemotherapy agents combined with cytotoxic anticancer agents. Resection for distant metastasis was performed in only 6 patients $(7.3 \%)$.

The median OS in the high serum CA19-9 concentration group ( $>370 \mathrm{U} / \mathrm{mL}$ ) was significantly worse than that of those with normal to elevated serum CA19-9 concentrations (0$370 \mathrm{U} / \mathrm{mL}$ ) (8.5 vs. 19.2 months, $p=0.0059$ ) (Fig. 1). Meanwhile, the median OS for those who 
Fig. 1. Kaplan-Meier curve of the impact of pretreatment serum CA19-9 concentration on overall survival ( $p=0.0059$; log-rank test). CA19-9, carbohydrate antigen 19-9.

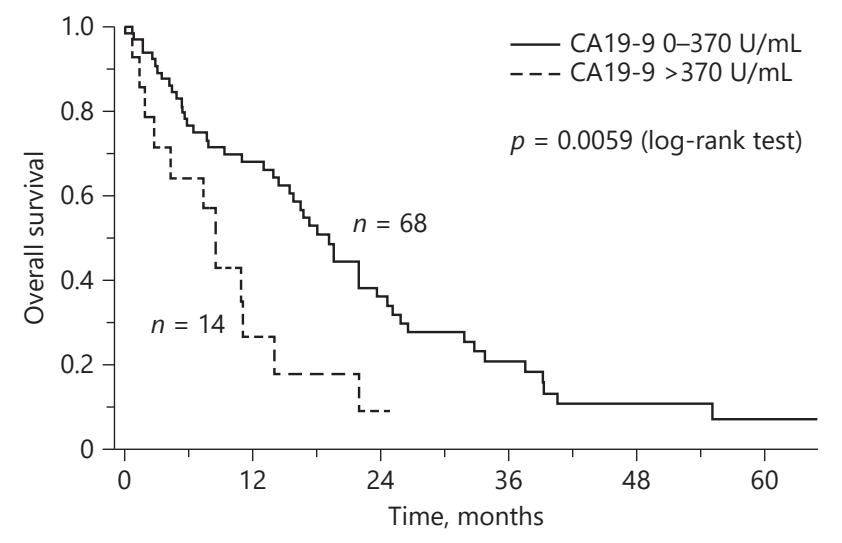

underwent resection in the primary tumor group was significantly higher than those in the nonresection group (18.1 vs. 11 months, $p=0.0122$ ). The median OS in the chemotherapy group was also significantly higher than that in the nonchemotherapy group (25.1 vs. 8.4 months, $p<0.0001$ ). OS was also significantly improved in the group of patients who underwent resection of distant metastasis compared with the nonresection group (39.4 vs. 11 months, $p=0.0022$ ). By contrast, no difference in OS was noted between the oral monotherapy group and the combination chemotherapy group ( $p=0.7976)$.

In univariate analysis, age ( $\geq 80$ years) $(p=0.014)$, PS of $1-3(p=0.028)$, and high pretreatment serum CA19-9 concentration $(p=0.014)$ were significant prognostic factors for poor OS (Table 2). By contrast, resection of the primary tumor $(p=0.024)$, chemotherapy $(p<0.0001)$, and resection of distant metastasis $(p=0.0005)$ were significant prognostic factors for favorable OS (Table 2).

Multivariate analysis also showed that high pretreatment serum CA19-9 concentration $(>370 \mathrm{U} / \mathrm{mL})$ was an independent prognostic factor for poor OS ( $p=0.01)$ (Table 2). Meanwhile, resection of the primary tumor $(p=0.033)$, chemotherapy $(p<0.0001)$, and resection of distant metastasis $(p=0.0008)$ were independent prognostic factors for favorable OS (Table 2).

\section{Discussion}

To our knowledge, this is one of the few retrospective studies on the prognostic factors in elderly patients ( $\geq 75$ years) with stage IV CRC. Our results showed that high pretreatment serum CA19-9 concentration was an independent prognostic factor for poor OS. By contrast, various treatments, including chemotherapy and resection of the primary and distant tumor, might improve the prognosis in elderly patients with stage IV CRC.

CA19-9 is an established tumor marker in CRC. Many studies have reported that CA19-9 is associated with distant metastasis and a prognostic factor in CRC [12-15]. Some researchers reported that preoperative serum CA19-9 concentration was a reliable predictive marker of tumor recurrence and prognosis in patients with stage IV CRC who had undergone curative resection $[16,17]$. Another study reported that preoperative high CA19-9 concentration was a poor prognosis factor in stage IV CRC with noncurable resection [18]. Moreover, postoperative CA19-9 at 3 months after curative resection in patients with stage IV CRC was reported to be a strong prognostic indicator of recurrence [19]. Although 
Table 2. Univariate and multivariate analysis for overall survival

\begin{tabular}{|c|c|c|c|c|c|c|}
\hline \multirow[t]{2}{*}{ Variables } & \multicolumn{3}{|c|}{ Univariate analysis } & \multicolumn{3}{|c|}{ Multivariate analysis } \\
\hline & OR & $95 \%$ CI & $p$ value & OR & $95 \% \mathrm{CI}$ & $p$ value \\
\hline \multicolumn{7}{|l|}{ Age } \\
\hline$\geq 80$ vs. $75-79$ years & 1.933 & $1.145-3.313$ & 0.014 & 1.079 & $0.572-2.037$ & 0.814 \\
\hline \multicolumn{7}{|l|}{ Sex } \\
\hline Male vs. female & 0.723 & $0.435-1.200$ & 0.209 & & & \\
\hline \multicolumn{7}{|l|}{ Primary tumor location } \\
\hline Right vs. left & 0.836 & $0.500-1.386$ & 0.489 & & & \\
\hline \multicolumn{7}{|l|}{ Performance status } \\
\hline $1-3$ vs. 0 & 1.931 & $1.076-3.367$ & 0.028 & 1.615 & $0.809-3.189$ & 0.172 \\
\hline \multicolumn{7}{|c|}{ Pretreatment serum CEA concentration } \\
\hline$>5$ vs. $0-5 \mathrm{ng} / \mathrm{mL}$ & 1.377 & $0.790-2.522$ & 0.266 & & & \\
\hline$>50$ vs. $0-50 \mathrm{ng} / \mathrm{mL}$ & 1.308 & $0.744-2.231$ & 0.342 & & & \\
\hline \multicolumn{7}{|c|}{ Pretreatment serum CA19-9 concentration } \\
\hline$>37$ vs. $0-37 \mathrm{U} / \mathrm{mL}$ & 1.378 & $0.824-2.298$ & 0.219 & & & \\
\hline$>370$ vs. $0-370 \mathrm{U} / \mathrm{mL}$ & 2.452 & $1.214-4.617$ & 0.014 & 2.828 & $1.304-5.820$ & 0.010 \\
\hline \multicolumn{7}{|c|}{ Resection of the primary tumor } \\
\hline Yes vs. no & 0.423 & $0.218-0.887$ & 0.024 & 0.378 & $0.160-0.924$ & 0.033 \\
\hline \multicolumn{7}{|l|}{ Emergency operation } \\
\hline Yes vs. no & 0.881 & $0.477-1.546$ & 0.668 & & & \\
\hline \multicolumn{7}{|l|}{ Lymphatic metastasis } \\
\hline N0 vs. $\mathrm{N}(+)$ & 1.460 & $0.726-2.728$ & 0.274 & & & \\
\hline \multicolumn{7}{|c|}{ Number of metastatic organs } \\
\hline M1b vs. M1a & 1.679 & $0.899-2.966$ & 0.100 & 1.187 & $0.538-2.583$ & 0.667 \\
\hline \multicolumn{7}{|l|}{ P3, H3, or PUL2 } \\
\hline Present vs. absent & 1.588 & $0.935-2.685$ & 0.087 & 1.079 & $0.546-2.053$ & 0.821 \\
\hline \multicolumn{7}{|l|}{ Chemotherapy } \\
\hline Yes vs. no & 0.331 & $0.192-0.568$ & $<0.0001$ & 0.209 & $0.105-0.402$ & $<0.0001$ \\
\hline \multicolumn{7}{|c|}{ Resection of metastatic tumor } \\
\hline Yes vs. no & 0.190 & $0.046-0.528$ & 0.0005 & 0.156 & $0.035-0.497$ & 0.0008 \\
\hline
\end{tabular}

Bold indicates statistical significance. CEA, carcinoembryonic antigen; CI, confidence interval; OR, odds ratio; CA19-9, carbohydrate antigen 19-9.

serum CA19-9 concentration might be associated with prognosis in stage IV CRC according to many reports, there were no studies concerning the association between serum CA19-9 concentration and OS in elderly patients with stage IV CRC. In this study, a serum CA19-9 concentration of $>37 \mathrm{U} / \mathrm{mL}$ was unrelated to OS; however, 1 out of 10 times the normal range at $>370 \mathrm{U} / \mathrm{mL}$ was a significant independent prognostic factor for OS. The median OS of the patients with stage IV CRC who had a serum CA19-9 concentration of $>370 \mathrm{U} / \mathrm{mL}$ was quite poor at 8.5 months. As such, these patients may no longer be eligible for aggressive treatments and be more suited to palliative care. Accordingly, serum CA19-9 concentration was an important biomarker for determining eligibility for aggressive treatment in elderly patients with stage IV CRC.

Many researchers have reported that curative resection might improve long-term survival in patients with stage IV CRC [20-22]. Recent retrospective studies on curatively resected stage IV CRC patients reported a 5-year OS rate of 52.2-57\% [22, 23]. Moreover, aggressive curative resection with perioperative chemotherapy might improve survival [22]. In general, curative resection and chemotherapy might be the optimal treatment strategy for middle-aged patients with stage IV CRC [9]. Some studies reported that resection 
of only the primary tumor improved OS or relapse-free survival in stage IV CRC [23-25]. In this study, the OS of the 66 patients $(80.5 \%)$ who underwent resection of the primary tumor was significantly longer than that of the patients who did not undergo primary tumor resection. Old age ( $\geq 75$ years) at the time of surgery for stage IV CRC was reported to be an independent risk factor for mortality during the first year after surgery [6]. Additionally, another study showed that a PS of 2-4 was an independent prognostic factor in patients with stage IV CRC aged $>80$ years [5]. In the current study, age and PS were poor prognostic factors in univariate analysis, but not in multivariate analysis. Considering the results of the current study and those of previous ones, resection of the primary tumor might be a beneficial treatment to improve OS in elderly patients with stage IV CRC. In addition, the patient's general characteristics, such as age and PS, should be considered when performing primary resection.

Despite the low rate of resection of distant metastasis in the current study at only $7.3 \%$, it significantly improved OS $(p=0.0022)$. In elderly patients, aggressive curative resection might yield the benefit of improving OS similar to that of younger patients. However, some studies reported that hepatic or pulmonary resection yielded higher mortality and complication rates in elderly compared to younger patients $[26,27]$. Therefore, the operative indications for distant metastasis must be carefully decided upon in elderly patients with stage IV CRC.

Similar to surgical resection, chemotherapy is also an important treatment in the management of patients with stage IV CRC [9]. It was reported that palliative chemotherapy in selected elderly patients ( $\geq 80$ years) with distant metastasis was considerably associated with improved OS, and oral 5-FU monotherapy in the palliative setting could be a reasonable option for elderly patients [5]. Kim [28] reported that chemotherapy in elderly patients should be individualized based on the nature of the disease, physiological or functional status, and patient's preference. In our study, 38 patients (46.3\%) received chemotherapy, and they had significantly longer survival than those who did not $(p<0.0001)$. This is similar to the result previously reported in another study [5]. Regarding the chemotherapy regimen, some studies reported that combination chemotherapy was not superior to monotherapy in terms of OS in elderly patients with metastatic CRC $[5,29]$. In the present study, OS was not significantly different between the patients who received mono-chemotherapy $(n=16,42.1 \%)$ and combination chemotherapy $(n=22,57.9 \%)$. We report results consistent with those of previous studies [5, 29]; mono-chemotherapy might be acceptable for these patients. However, randomized prospective studies are still needed to further determine the optimal chemotherapy regimen for elderly patients with stage IV CRC.

This study had some limitations, including its retrospective and single-institution design as well as the relatively small number of patients analyzed. All patients who were analyzed underwent surgical treatments including palliative surgery. The patients who underwent primary resection or chemotherapy might have been in a relatively better condition compared to the patients who did not undergo treatment. A large-scale or randomized study is necessary to confirm our results.

\section{Conclusions}

Our study demonstrated that a high pretreatment serum CA19-9 concentration ( $>370 \mathrm{U} /$ $\mathrm{mL}$ ) was a reliable predictive factor for poor prognosis in elderly patients with stage IV CRC, and aggressive treatments should be performed carefully in these patients. Moreover, various treatments, including surgery and chemotherapy, might improve OS in elderly patients with stage IV CRC. 


\section{Statement of Ethics}

This study was approved by our hospital's review committee (approval No. 17H100). Informed consent for the use of the clinical and pathological data of all patients was obtained via opt-out presented on our hospital's homepage.

\section{Disclosure Statement}

The authors declare that they have no competing interests. No financial support was received.

\section{References}

1 Torre LA, Bray F, Siegel RL, Ferlay J, Lortet-Tieulent J, Jemal A. Global cancer statistics, 2012. CA Cancer J Clin. 2015 Mar;65(2):87-108.

2 Siegel R, Desantis C, Jemal A. Colorectal cancer statistics, 2014. CA Cancer J Clin. 2014 Mar-Apr;64(2):104-17.

3 Etzioni DA, Beart RW Jr, Madoff RD, Ault GT. Impact of the aging population on the demand for colorectal procedures. Dis Colon Rectum. 2009 Apr;52(4):583-90.

4 Zhao M, Liu H, Tang Y, Meng X, Yu J, Wang Q, et al. Clinicopathologic features and prognostic factors for patients with colorectal cancer who are 75 years and older. Oncotarget. 2017 Sep;8(45):80002-11.

5 Jung YH, Kim JY, Jang YN, Yoo SH, Kim GH, Lee KM, et al. Clinical characteristics and treatment propensity in elderly patients aged over 80 years with colorectal cancer. Korean J Intern Med. 2018 Nov;33(6):1182-93.

6 Kornmann VNN, van Vugt JLA, Smits AB, van Ramshorst B, Boerma D. The first year after colorectal surgery in the elderly. Ann Coloproctol. 2017 Aug;33(4):134-8.

7 Temple LK, Hsieh L, Wong WD, Saltz L, Schrag D. Use of surgery among elderly patients with stage IV colorectal cancer. J Clin Oncol. 2004 Sep;22(17):3475-84.

8 Yamano T, Yamauchi S, Kimura K, Babaya A, Hamanaka M, Kobayashi M, et al.; Japanese Study Group for Postoperative Follow-up of Colorectal Cancer: Influence of age and comorbidity on prognosis and application of adjuvant chemotherapy in elderly Japanese patients with colorectal cancer: A retrospective multicentre study. Eur J Cancer. 2017 Aug; 81:90-101.

9 Watanabe T, Muro K, Ajioka Y, Hashiguchi Y, Ito Y, Saito Y, et al.; Japanese Society for Cancer of the Colon and Rectum. Japanese Society for Cancer of the Colon and Rectum (JSCCR) guidelines 2016 for the treatment of colorectal cancer. Int J Clin Oncol. 2018 Feb;23(1):1-34.

10 National Comprehensive Cancer Network. NCCN clinical practice guideline in oncology. Colon cancer version 2. Available via: http://www.nccn.org/professionals/physician_gls/pdf/colon.pdf. Accessed April 2018.

11 Japan Society for Cancer of the Colon and Rectum. Japanese Classification of Colorectal Carcinoma. 8th ed. Tokyo: Kanehara \& Co. Ltd; 2013.

12 Stojkovic Lalosevic M, Stankovic S, Stojkovic M, Markovic V, Dimitrijevic I, Lalosevic J, et al. Can preoperative CEA and CA19-9 serum concentrations suggest metastatic disease in colorectal cancer patients? Hell J Nucl Med. 2017 Jan-Apr;20(1):41-5.

13 Yu Z, Chen Z, Wu J, Li Z, Wu Y. Prognostic value of pretreatment serum carbohydrate antigen 19-9 level in patients with colorectal cancer: A meta-analysis. PLoS One. 2017 Nov;12(11):e0188139.

14 Takakura Y, Ikeda S, Imaoka Y, Urushihara T, Itamoto T. An elevated preoperative serum carbohydrate antigen 19-9 level is a significant predictor for peritoneal dissemination and poor survival in colorectal cancer. Colorectal Dis. 2015 May; 17(5):417-25.

15 Yang XQ, Chen C, Wang FB, Peng CW, Li Y. Preoperative serum carcinoembryonic antigen, carbohydrate antigen 19-9 and carbohydrate antigen 125 as prognostic factors for recurrence-free survival in colorectal cancer. Asian Pac J Cancer Prev. 2011;12(5):1251-6.

16 Ozawa T, Ishihara S, Kawai K, Nozawa H, Yamaguchi H, Kitayama J, et al. Prognostic significance of preoperative serum carbohydrate antigen 19-9 in patients with stage IV colorectal cancer. Clin Colorectal Cancer. 2016 Dec;15(4):e157-63.

17 Lu Z, Peng J, Wang Z, Pan Z, Yuan Y, Wan D, et al. High preoperative serum CA19-9 level is predictive of poor prognosis for patients with colorectal liver oligometastases undergoing hepatic resection. Med Oncol. 2016 Nov;33(11):121.

18 Katoh H, Yamashita K, Kokuba Y, Satoh T, Ozawa H, Hatate K, et al. Surgical resection of stage IV colorectal cancer and prognosis. World J Surg. 2008 Jun;32(6):1130-7.

19 Abe S, Kawai K, Ishihara S, Nozawa H, Hata K, Kiyomatsu T, et al. Prognostic impact of carcinoembryonic antigen and carbohydrate antigen 19-9 in stage IV colorectal cancer patients after R0 resection. J Surg Res. 2016 Oct;205(2):384-92.

20 Murata S, Moriya Y, Akasu T, Fujita S, Sugihara K. Resection of both hepatic and pulmonary metastases in patients with colorectal carcinoma. Cancer. 1998 Sep;83(6):1086-93. 
21 Kobayashi K, Kawamura M, Ishihara T. Surgical treatment for both pulmonary and hepatic metastases from colorectal cancer. J Thorac Cardiovasc Surg. 1999 Dec;118(6):1090-6.

22 Mukai T, Uehara K, Aiba T, Nakamura H, Ebata T, Nagino M. Outcome of stage IV patients with colorectal cancer treated in a single institution: what is the key to the long-term survival? J Anus Rectum Colon. 2018;2(1): $16-24$.

23 Huh JW, Lee WY, Park YA, Cho YB, Yun SH, Kim HC, et al. Prognostic factors associated with primary cancer in curatively resected stage IV colorectal cancer. J Cancer Res Clin Oncol. 2014 Mar;140(3):435-41.

24 Venderbosch S, de Wilt JH, Teerenstra S, Loosveld OJ, van Bochove A, Sinnige HA, et al. Prognostic value of resection of primary tumor in patients with stage IV colorectal cancer: retrospective analysis of two randomized studies and a review of the literature. Ann Surg Oncol. 2011 Nov;18(12):3252-60.

25 't Lam-Boer J, Van der Geest LG, Verhoef C, Elferink ME, Koopman M, de Wilt JH. Palliative resection of the primary tumor is associated with improved overall survival in incurable stage IV colorectal cancer: A nationwide population-based propensity-score adjusted study in the Netherlands. Int J Cancer. 2016 Nov; 139(9):2082-94.

26 Phan K, An VV, Ha H, Phan S, Lam V, Pleass H. Hepatic resection for malignant liver tumours in the elderly: a systematic review and meta-analysis. ANZ J Surg. 2015 Nov;85(11):815-22.

27 Stamenovic D, Messerschmidt A, Schneider T. Surgery for lung tumors in the elderly: A retrospective cohort study on the influence of advanced age (over 80 years) on the development of complications by using a multivariate risk model. Int J Surg. 2018 Apr;52:141-8.

28 Kim JH. Chemotherapy for colorectal cancer in the elderly. World J Gastroenterol. 2015 May;21(17):5158-66.

29 Aparicio T, Lavau-Denes S, Phelip JM, Maillard E, Jouve JL, Gargot D, et al.; FFCD investigators. Randomized phase III trial in elderly patients comparing LV5FU2 with or without irinotecan for first-line treatment of metastatic colorectal cancer (FFCD 2001-02). Ann Oncol. 2016 Jan;27(1):121-7. 